\title{
INVESTIGATIONS INTO FLOUR MIXES OF TRITICUM MONOCOCCUM AND TRITICUM SPELTA
}

\author{
KATALIN KÓcZÁn-MANNINGER ${ }^{* 1}$ AND KATALIN BADAK-KERTI ${ }^{1}$ \\ ${ }^{1}$ Department of Grain and Industrial Plant Processing, Szent István University, Villányi út 29-43, Budapest, \\ 1118, HUNGARY
}

\begin{abstract}
Bread samples were made using flour mixes of Triticum monococcum (Tr. monococcum) and Triticum spelta (Tr. spelta). They were tested for their rheological behaviour over the first 3 days of storage at room temperature, and for their characteristics based on a Hungarian Standard. Parameters were set such as the volume of the baked product, baking loss, crumb characteristics and elasticity of crumbs. The behaviour of flour from einkorn wheat is different to that of $T r$. spelta. The properties of the tested flour mixes measured by a farinograph show that $T r$. spelta produces an acceptable dough, on the other hand, the dough of Tr. monococcum develops quickly but is very unstable so weakens within minutes of being kneaded. This also suggests that doughs composed of einkorn wheat flour require a different type of kneading than those of Tr. spelta (or Tr. aestivum, also referred to as common wheat) flours. Breads composed of Tr. spelta were comparable with those made with Tr. aestivum, the crumb elasticity was above $90 \%$ on the day of baking, which indicates high quality. The Tr. monococcum breads, however, were of low grade: the volume of the breads decreased by increasing the ratio of Tr. monococcum to Tr. spelta and the elasticity reduced to unacceptable levels (less than $60 \%$ ). It should be mentioned that the grading was based on breads made purely from Tr. aestivum flours.
\end{abstract}

Keywords: spelt, einkorn, bread, texture analysis

\section{Introduction}

As a result of the increasing number of cases of celiac disease and allergies, as well as the growing popularity of conscious nutrition, interest in older varieties of wheat is once again on the rise. In general, consumers think that these species of wheat are potentially less immunogenic than their modern equivalents. The manufacturing properties of doughs produced from ancient varieties of wheat are much weaker than those of common wheat. In order to obtain good quality bakery products, it may be necessary to use mixtures of flours from different varieties.

In our research, the properties of the flour of einkorn and spelt wheats in addition to breads that consist of different proportions of these flours were prepared and investigated. During measurements, attempts were made to determine whether these wheat species - which are in theory suitable for baking bread - could improve the baking performance or whether a significant difference exists between the characteristics of the finished products of various compositions.

Crossing more modern varieties results in higher yields, greater resistance, more uniform ripening times and higher gluten contents. Although these breeding procedures facilitated processing, the genetic diversity and nutritional value decreased significantly which virtually

\footnotetext{
*Correspondence: koczan.gyorgyne@etk.szie.hu
}

resulted in the total displacement of indigenous species $[1,2]$. One reason for this is that $T r$. monococcum was consumed primarily as a mush or simply cooked; these methods did not require proofing, which was originally used in ancient Egypt during bread baking [3]. Bread made from spelt flour is also of lower quality than that of common wheat, both in terms of specific volume and crumb structure [4].

According to previous research, spelt wheat flour produces less stable and elastic but stickier dough than plain flour. Due to its sticky and soft nature after kneading, it is difficult to handle $[5,6]$. Breads made from einkorn flour exhibit a wide range of possible specific volumes, ranging from very low to high. Although only a few subtypes are suitable for making breads, most versions are suitable for preparing pasta or biscuits [7], or utilisation for special purposes like fermentation processes [8].

The first phase of the investigations concerned the quality of the gluten, followed by the preparation and testing of loaves of bread. The main question concerned how the blends of flours of these species of wheat influence the quality of the final products. 


\section{Experimental}

\subsection{Samples and Measurements}

Triticum monococcum (einkorn) and Triticum spelta wheat flours were manufactured by Szabó Hengermalom Kft. using conventional technology and contained no additives or bread improvers. For the measurements fine flours were used, i.e. small grain particles with low bran content, to ensure they contained only a negligible amount of outer shell.

The determination of wet gluten content was performed according to a standard using the Glutomatic System. After gluten washing, a gluten index was also calculated using a gluten centrifuge.

The moisture content was determined by a Sartorius moisture analyser. The uniformly dispersed sample of 2.5 $\mathrm{g}$ was dried at $105^{\circ} \mathrm{C}$ to a constant weight (which has not changed for 20 seconds more than $1 \mathrm{mg}$ ). The change in mass could be deduced from the moisture content of the whole test substance.

The determination of water absorption was conducted by a Brabender farinograph in accordance with a Hungarian standard (MSZ 6369-6:2013) in duplicates, followed by further experimentation using a baking test (MSZ 6369-8:1988).

The volume of the bread samples was measured by placing a loaf in a container of known volume and pouring in a known quantity of mustard seeds around the loaf until the container was full. By measuring the amount of seeds remaining once the container was full, the volume of the loaf could be calculated.

The quality of the bread texture was evaluated by a TA.XTplus texture analyser (Stable Micro Systems, Surrey, UK), following a modified American Association of Cereal Chemists (AACC) International approved method (74-09) and expressed as crumb firmness (force, $1 / \mathrm{g}$ ) and relative elasticity (\%). A $40 \%$ compression of a $25 \mathrm{~mm}$ thick sample was achieved, following a resting time of 30 seconds (at the same compression depth) and then the measuring head was slowly lifted and the springiness of the sample calculated. Thus, it was a "measure of force in compression" test using an AACC 36mm-diameter cylinder probe with radius $(\mathrm{P} / 36 \mathrm{R})$. The analyser was set at a 'return to start' cycle with a pre-test speed of $1 \mathrm{~mm} \mathrm{~s}^{-1}$, a test speed of $0.5 \mathrm{~mm} \mathrm{~s}^{-1}$, a post-test speed of $10 \mathrm{~mm} \mathrm{~s}^{-1}$ and a pre-defined percentage $(40 \%)$ of the original sample height. The relative elasticity was calculated from the difference between the original height and the height to which the sample recovered (after pressing and releasing the pressure).

Measurements were conducted in triplicates. Statistical evaluations were carried out using ANOVA (analysis of variance) tests in Excel.

Bread samples were stored at room temperature in plastic bags. Texture measurements were taken on the day of baking after the bread had been cooled to room temperature (Day 0) and on the following 2 days, namely Days 1 and 2 .
Table 1: Composition of the samples (\%)

\begin{tabular}{lllllll}
\hline & $100 \mathrm{~A}$ & $80 \mathrm{~A}$ & $60 \mathrm{~A}$ & $40 \mathrm{~A}$ & $20 \mathrm{~A}$ & $100 \mathrm{~T}$ \\
\hline $\begin{array}{l}\text { Tr. monoc. } \\
\text { (A) } \%\end{array}$ & 100 & 80 & 60 & 40 & 20 & 0 \\
\hline $\begin{array}{l}\text { Tr. spelta } \\
\text { (T) } \%\end{array}$ & 0 & 20 & 40 & 60 & 80 & 100 \\
\hline Water \% & 57 & 62 & 64 & 65.4 & 65.8 & 71 \\
\hline Yeast \% & 4 & 4 & 4 & 4 & 4 & 4 \\
\hline Salt \% & 1.2 & 1.2 & 1.2 & 1.2 & 1.2 & 1.2 \\
\hline
\end{tabular}

The ingredients consisted of $250 \mathrm{~g}$ of flour, $10 \mathrm{~g}$ of yeast and $3 \mathrm{~g}$ of salt, the only variable parameter was the amount of water used to make the dough. Initially, the dough consisted of approximately $60 \%(150 \mathrm{ml})$ water based on the weight of the flour, and the amount of water was increased to form a homogeneous dough. The final compositions are shown in Table 1.

\section{Results and Discussion}

\subsection{Experiments}

In the case of the einkorn flour, gluten washing was ineffective as it could not be washed out. After the mixing phase, a yellowish substance remained on the bottom of the washer. In the case of spelt flour, gluten tests could be conducted without any problems.

The wet gluten content of the Tr. spelta flour was $46.73 \%$. According to the Hungarian regulations bread wheat flours must have a minimum wet gluten content of $28 \%$ and for wheat flours used to improve the baking quality a minimum of $34 \%$. Bakers consider a gluten content in excess of $30 \%$ to be good. The wet gluten content of the spelt flour examined is well above this value, but other factors are also taken into account to determine the quality of flour.

The gluten index, a measure of gluten quality, of spelt flour was $45.73 \%$. A value of between 60 and $90 \%$ is considered to be ideal, below $60 \%$ weak and in excess of $90 \%$ too strong. Thus, the gluten quality of the spelt flour was clearly weak.

The gluten quality calculated from the results of the farinograph tests for spelt flour was $98 \%$ which is acceptable but does not fully reflect the quality of the flour. Although the kneading and stability times of the doughs fell within the range of expected values, the planimetric area was greater due to the degree of softening. Thus, the quality score obtained by Hankóczy's evaluation method was smaller. The farinogram of spelt flour more closely resembles a flour of medium quality (Fig. 1).

This is especially true for the Tr. monococcum flour. It reaches its maximum consistency very quickly; the top of the curve barely exceeds the consistency line (500 BU - Brabender Units). The degree of softening is enormous, as is reflected well by the large planimetric area. The qualitative value assigned to the curve is very low (Fig. 2). 


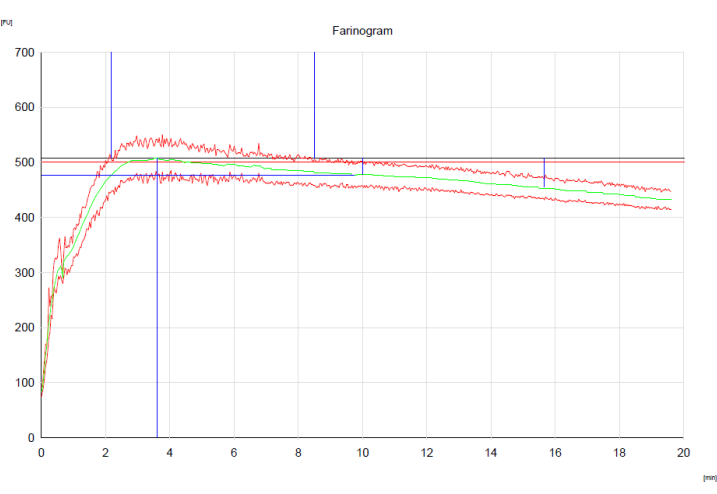

Figure 1: Farinogram of Triticum spelta flour.

A direct correlation was identified between the volume of the bread samples and the amount of spelt flour in the flour blend (Fig. 3). This is in accordance with the gluten quality of the flour blends, as is seen from the results of the farinograph measurements.

The crumb hardness of the bread samples is shown in Fig. 4. As the samples started to age the compression force increased. By examining the initial and final forces (measures of crumb hardness), it can be stated that sample $60 \mathrm{~A}$ showed the best results. In this case, the force increased by $29 \%$ between Day 0 and Day 2. For samples containing less einkorn flour the crumbs seemed to be softer and the relative increase in hardness during storage less (when values on Day 2 were compared to those on Day 0). Even though sample 80A was initially even softer than $60 \mathrm{~A}$, by the end of Day 2 it needed 1.7 times the force to compress it. An explanation of this phenomenon can also be given with regard to the different compositions of the starch molecules in einkorn flour compared to those in spelt flour. The staling of bread is related to the crystallization processes of starch molecules.

Significant differences between samples consisting of $100 \%$ spelt flour and those of $20 \%$ einkorn flour mixed with $80 \%$ spelt flour were shown by the results. The increase in crumb hardness during storage resulted in significant differences in all samples of identical compositions.

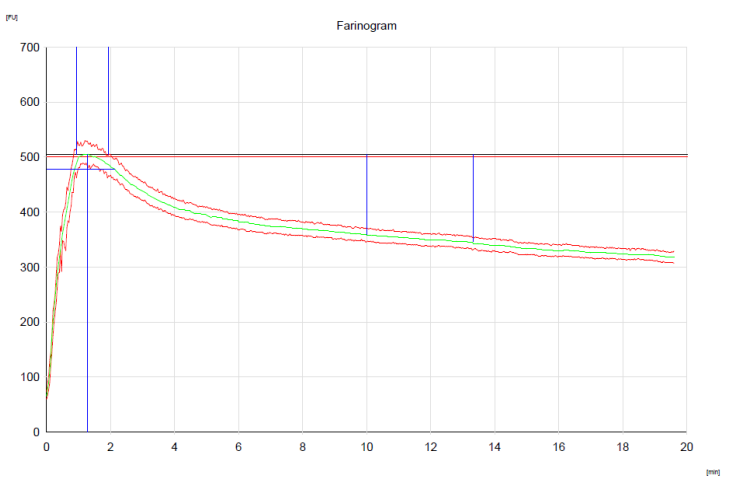

Figure 2: Farinogram of Triticum monococcum flour

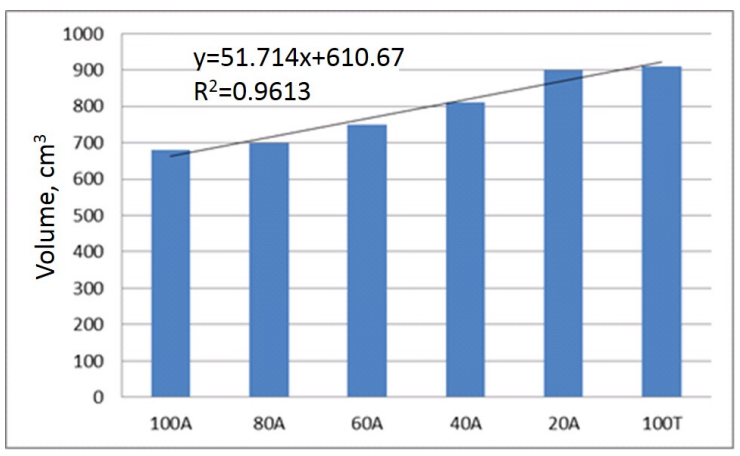

Figure 3: Volume of bread samples (A - einkorn flour, $\mathrm{T}$ - spelt flour; the numbers are the percentages of einkorn flour in the flour blend)

The elasticity of the bread crumbs increased as the amount of spelt flour increased in the flour blend (Fig. 5). This tendency persisted during storage as well. The slight increase in the elasticity of the bread composed of $100 \%$ Triticum monococcum flour was probably due to improper handling of the samples, i.e. improper cooling before being packed, although it is questionable whether any moisture originating from the headspace of the packaging could cause such a change.

Taking into account that the results obtained could be derived from measurement and/or calculation errors, it may be worthwhile to consider the role of the chemical structure of einkorn flour during the baking process, and its effect on the elasticity during further targeted experiments.

By using a rating system for the Tr. aestivum flours, the bread samples can be classified. Although the same judgment about the "marketability" of the bread samples cannot be made for breads based on these special types of flour, trends can clearly be observed. By adding more einkorn flour to the flour blends, the "quality" of the crumb structure decreased.

Most of the samples did not achieve an elasticity of 80 $\%$ meaning that they did not return to $80 \%$ of their original height after compression. With these values, most of the breads fall into the non-marketable category. Elastic-

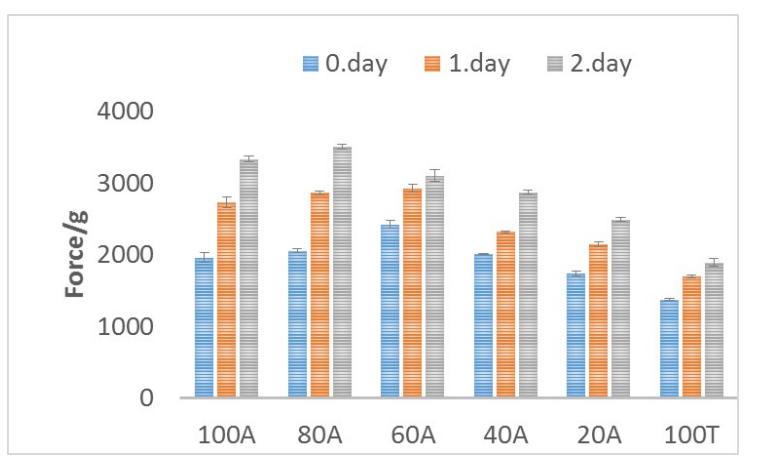

Figure 4: Crumb hardness (Force, $1 / \mathrm{g}$ ) as a function of different flour compositions over 3 days 


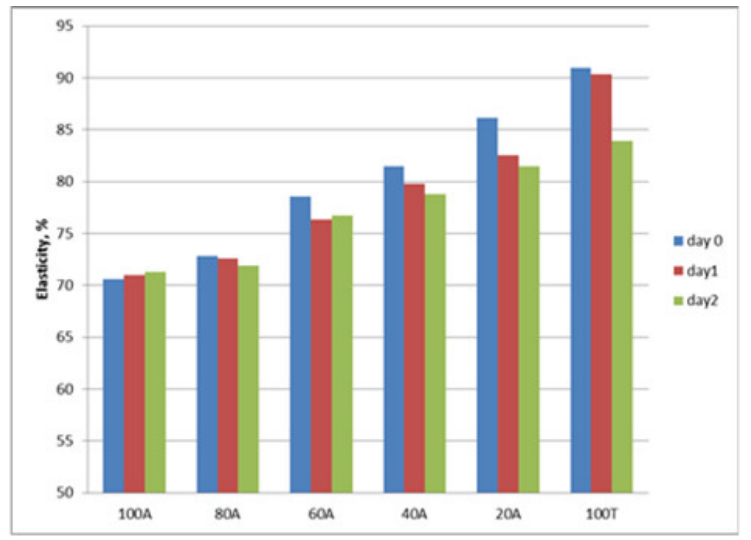

Figure 5: Change in the elasticity of the bread samples during storage at room temperature

ities of between 90 and $95 \%$ are indicative of good quality breads. Such values were only achieved when $100 \%$ Triticum spelta flour was used. After 2 days of storage at room temperature, the crumbs of $100 \%$ spelt flour bread degraded to an average quality.

\section{Conclusion}

The purpose of our investigations was to examine the quality of flours from varieties of ancient wheats.

Gluten could not be washed out of einkorn samples and the wet gluten content of Tr. spelta was also very low. Farinograph measurements revealed that when only einkorn flour is used, the dough forms very fast but is very soft and almost completely unstable.

By mixing einkorn and spelt flours bread can be made, however, an acceptable ratio would not exceed $20 \%$ of einkorn to $80 \%$ of $T r$ spelta flour. With this flour blend, the resulting bread volume is comparable to the accepted low values of bread composed of $100 \%$ spelt flour. The hardness and elasticity of the bread crumbs already changed significantly at the lowest mixing ratios.

Further studies on the sensory characteristics of these breads and consumer tests are needed before deciding on the use of flour blends of Triticum monococcum and
Triticum spelta in the absence of any addition of Triticum aestivum flour.

\section{REFERENCES}

[1] Draskovics, M. R.: Seed plants (Spermatophyta) In: Turcsányi, G. (ed.) Agricultural botany, Mezőgazdasági Szaktudási Kiadó, Budapest, Hungary, 2000 pp 363-365 ISBN: 9633563593

[2] Dinu, M.; Whittaker, A.; Paglia, G.; Benedettelli, S.; Sofi, F.: Ancient wheat species and human health: biochemical and clinical implications. J. Nutr. Biochem., 2018 52, 1-9 DOI: 10.1016/j.jnutbio.2017.09.001

[3] Brandolini, A.; Hidalgo, A.: Chapter 8: Einkorn (Triticum monococcum) Flour and Bread in Flour and Breads and their Fortification In: Preedy V. R.; Watson R. R.; Patel V. B.: Health and Disease Prevention, Academic Press/Elsevier, UK, 2011 pp 7988 ISBN: 978-0-12-380886-8

[4] Abdel-Aal, E-S. M.; Hucl, P.; Sosulski, W.; Bhirud, P. R.: Kernel, milling and baking properties of spring-type spelt and einkorn wheats. J. Cereal Sci., 1997 26, 363-370 DOI: 10.1006/jcrs.1997.0139

[5] Callejo, M. J.; Vargas-Kostiuk, M. E., RodríguezQuijano, M.: Selection, training and validation process of a sensory panel for bread analysis: Influence of cultivar on the quality of breads made from common wheat and spelt wheat. J. Cereal Sci., 2015 61, 55-62 DOI: 10.1016/ j.jcs.2014.09.008

[6] Frakolaki, G.; Giannou, V.; Topakas, E.; Tzia, C.: Chemical characterization and breadmaking potential of spelt versus wheat flour. J. Cereal Sci., 2017 79, 50-56 DOI: 10.1016/j.jcs.2014.09.008

[7] Hidalgo, A., Brandolini, A.: Lipoxygenase activity in wholemeal flours from Triticum monococcum, Triticum turgidum and Triticum aestivum. Food Chem., 2012 131, 1499-1503 DOI: 10.1016/j.foodchem.2011.09.132

[8] Hetényi, K.; Németh, Á.; Sevella, A.: Examination of medium supplementation for lactic acid fermentation. Hung. J. Ind. Chem., 2008 36(1-2) 49-53 